\title{
ODONTOID FRACTURES
}

\author{
A RATIONAL APPROACH TO TREATMENT
}

M. D. RYAN, T. K. F. TAYLOR

\section{From the Department of Orthopaedics and Traumatic Surgery, The Royal North Shore Hospital of Sydney, and The University of Sydney}

Twenty-three adults with fractures of the odontoid process are reviewed. The possible reasons for the high rate of non-union in reported series are considered: these include the type of fracture, its displacement, the presence of a gap at the fracture site, imperfect reduction and inadequate immobilisation.

Type 2 fractures (at the base of the odontoid process) are the commonest and also the most liable to nonunion. In their treatment, reduction is important; as seen in the lateral radiograph at least two-thirds of the fracture surfaces should be in contact. Skull traction is not advised and halo-cast fixation is the treatment of choice; with this method 87.5 per cent of recent odontoid fractures united.

In the field of spinal injuries, fractures of the odontoid are something of an enigma, with reported rates of non-union as high as 62 per cent (Schatzker, Rorabeck and Waddell 1971). The exact mechanisms of injury are poorly understood and experimental studies on cadavers have not fully elucidated the pathomechanics. Odontoid fractures are not common and their incidence in reported series of cervical fractures varies from 7 to 14 per cent (Crooks and Birkett 1944; Amyes and Anderson 1956; Nachemson 1959; Böhler 1965). The purpose of this paper is to present a plan of management based on anatomical factors, which we consider have not previously received due attention.

\section{CLASSIFICATION AND MECHANISM OF INJURY}

Anderson and D'Alonzo (1974) divided odontoid fractures into three types (Fig. 1); we have found this classification to be of practical value in predicting the likelihood of non-union.

With regard to the mechanism of injury, many previous authors have concluded that odontoid fractures were almost always due to a blow on the head and not the neck (Jefferson 1920, 1927; Plaut 1938). Schatzker, Rorabeck and Waddell (1971) were of the opinion that the dens may break as a result of a combination of shearing and avulsion forces, and that displacement was the result of the former. More recently, Mouradian et al. (1978), in a study of isolated adult cadaveric spines, produced a Type 3 fracture from a flexion injury, whilst a Type 2 fracture was produced by a lateral loading injury. The latter, however, lacks clinical correlation, and they suggested that, in vivo, the probable mechanism was a frontotemporal impact on the rotated cervical spine
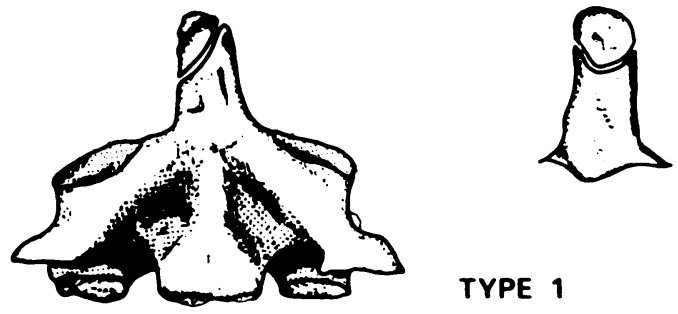

TYPE 1
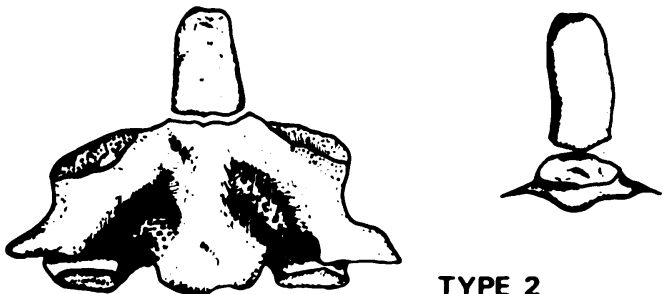

TYPE 2

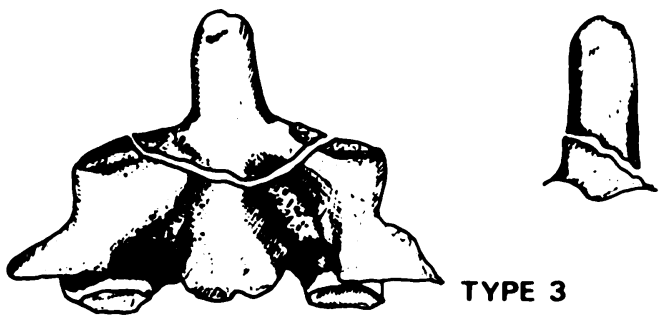

Fig. 1

Anderson and D'Alonzo's classification 
producing ipsilateral loading on the lateral mass of $\mathrm{Cl}$ and shearing the peg from the body of the axis. Such an injury has not been produced experimentally in cadavers.

\section{FACTORS WHICH CONTRIBUTE TO NON- UNION}

The factors discussed below have all been considered in determining the high rate of non-union.

Fracture type. Type 1 fractures always unite and Type 3 fractures nearly always. It is Type 2 fractures, the commonest variety, which have such a high rate of nonunion. Anderson and D'Alonzo (1974) give the following rates of non-union with conservative treatment: Type 1 , nil; Type 2, 36 per cent; and Type 3, seven per cent.

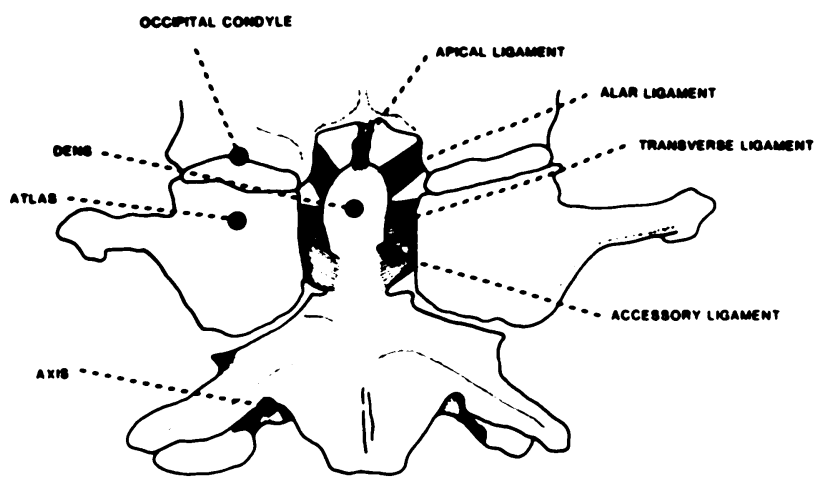

Fig. 2

Ligamentous attachments.

Ligamentous attachments. The ligamentous attachments of the odontoid process are important in the pathomechanics of fracture (Fig. 2). The apical ligament and the paired alar ligaments fan out from the superior aspect and the sides of the dens respectively; the former to blend with the tectorial membrane and the latter to attach to the anterior lip of the foramen magnum and the occipital zondyles. The strong transverse ligament is attached to the anteromedial aspect of the lateral masses of the atlas and curves posteriorly around the dens, from which it is separated by a small synovial bursa. Schatzker et al. (1971) have also demonstrated a ligamentous band which arises in conjunction with the transverse ligament and passes directly to the lateral aspect of the dens immediately above its base, at the level of the medial margin of the apophysial articulations.

All these ligaments are attached to the separated proximal fragment in Type 2 fractures (Fig. 3). Consequently, in these, the skull, atlas and dens move as a single unit, and displacement on the body of the axis is restricted only by the capsules of the atlanto-axial apophysial joints (which must be lax to allow rotation) and by the cervical musculature. The alar ligaments, in particular, transmit twisting movements to the cephalad fragment causing rotary displacement. Clearly Type 2 fractures are intrinsically unstable and prone to displacement. The ligamentous attachments also explain why
Type 1 fractures are inherently stable (Fig. 4). Type 3 fractures are reasonably stable, but for different reasons: the interface between the fractured surface is large, and the causal force is usually flexion (Mouradian et al. 1978) with consequent impaction.

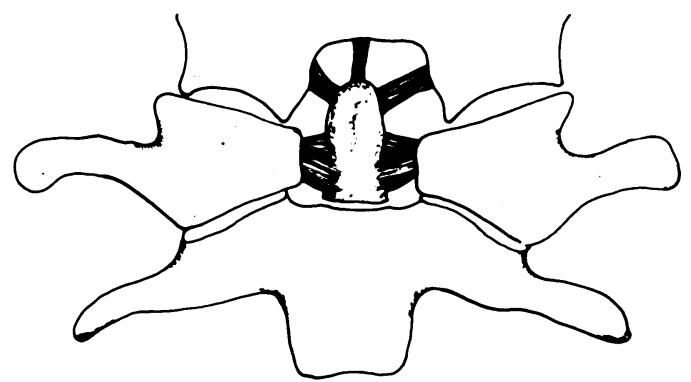

Fig. 3

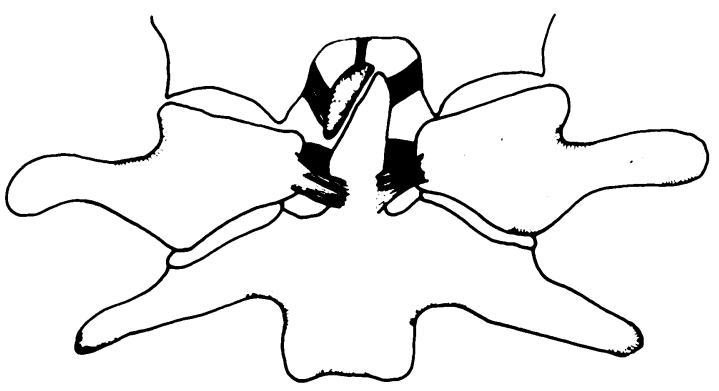

Fig. 4

Figure 3-Type 2 fracture, where there is no hold on the distal fragment. Figure 4-Type 1 fracture, in which the fragments are controlled by the ligaments.

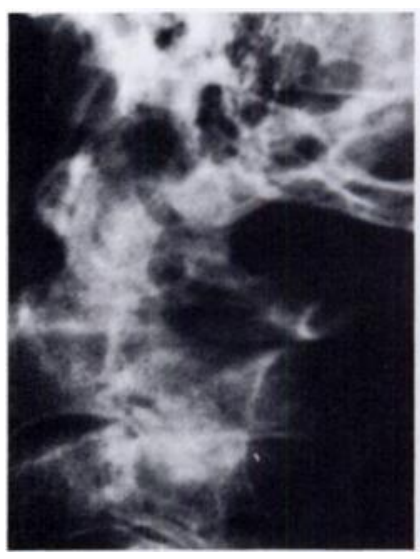

Fig. 5

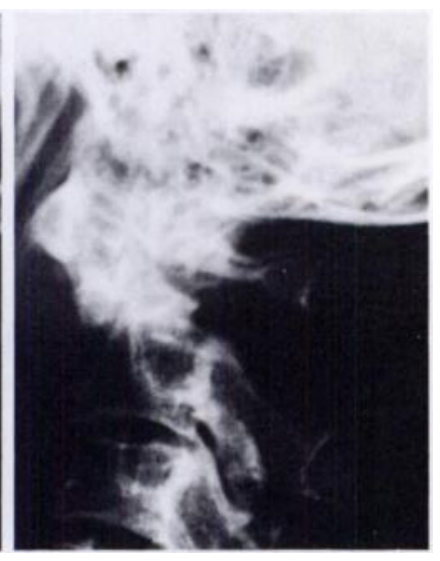

Fig. 6
Radiographs in extension and flexion. Note the alteration from posterior to anterior displacement.

Displacement. Marked displacement of Type 2 fractures, particularly posteriorly, has been correlated with a high rate of non-union (Schatzker et al. 1971). The initial direction of displacement is not necessarily significant; manipulation or accidental movement may easily convert posterior to anterior displacement. Two such conversions were noted in the present series and Figures 5 and 6 illustrate one of these.

Skull traction. The anatomical factors which allow displacement of Type 2 fractures also tend to cause distraction at the fracture when skull traction is applied 
(Fig. 7). The deleterious effects of traction are best seen in the child with a fractured odontoid. Here skull traction can produce wide displacement through the synchondrosis between the dens and the body of the axis (Fig. 8). We contend that cervical traction has no place in the treatment of fractures of the odontoid, and may indeed be positively harmful.

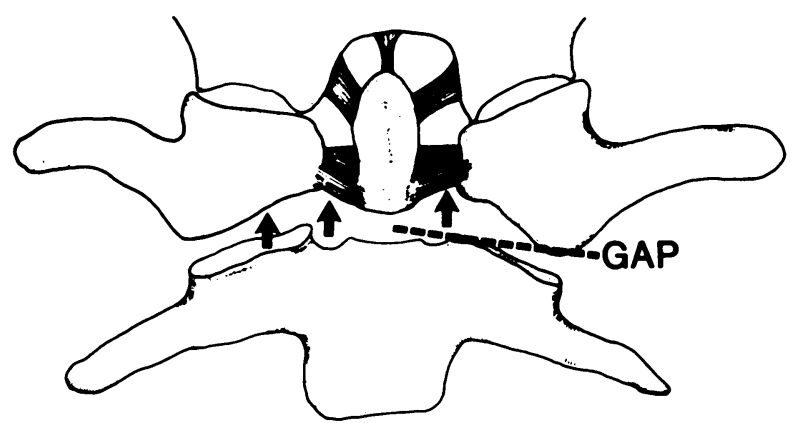

Fig. 7

The effect of skull traction.

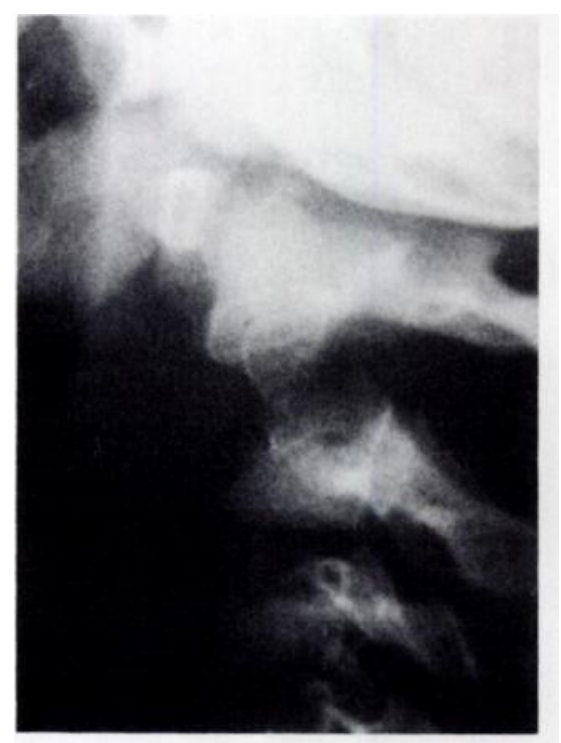

Fig. 8

Wide separation through the synchondrosis produced by traction in a child.

The distracting effect of skull traction has attracted little attention in the literature though it is clearly important, for Schatzker, Rorabeck and Waddell (1975) have demonstrated that when a gap was produced in odontoid osteotomies in dogs, non-union occurred. It is widely accepted that in fractures of cancellous bone, a gap at the fracture site may foreshadow non-union; a familiar example is a fracture of the waist of the carpal scaphoid. The same principle holds for odontoid fractures and in seems logical to try to avoid producing a gap, and to immobilise these fractures rigidly.

The adequacy of reduction. One essential feature for successful treatment of fractures in cancellous bone is adequate reduction. The base of the odontoid process is essentially circular in cross-section, and in the adult the cross-sectional area is approximately 80 square millimetres. In displaced Type 2 fractures the area of contact between the fragments is less than a superficial appraisal of the radiographs might suggest, because the interface consists of two overlapping circles. Figure 9 shows the area of contact at the fracture site in relation to differing amounts of displacement in the anteroposterior and lateral views.
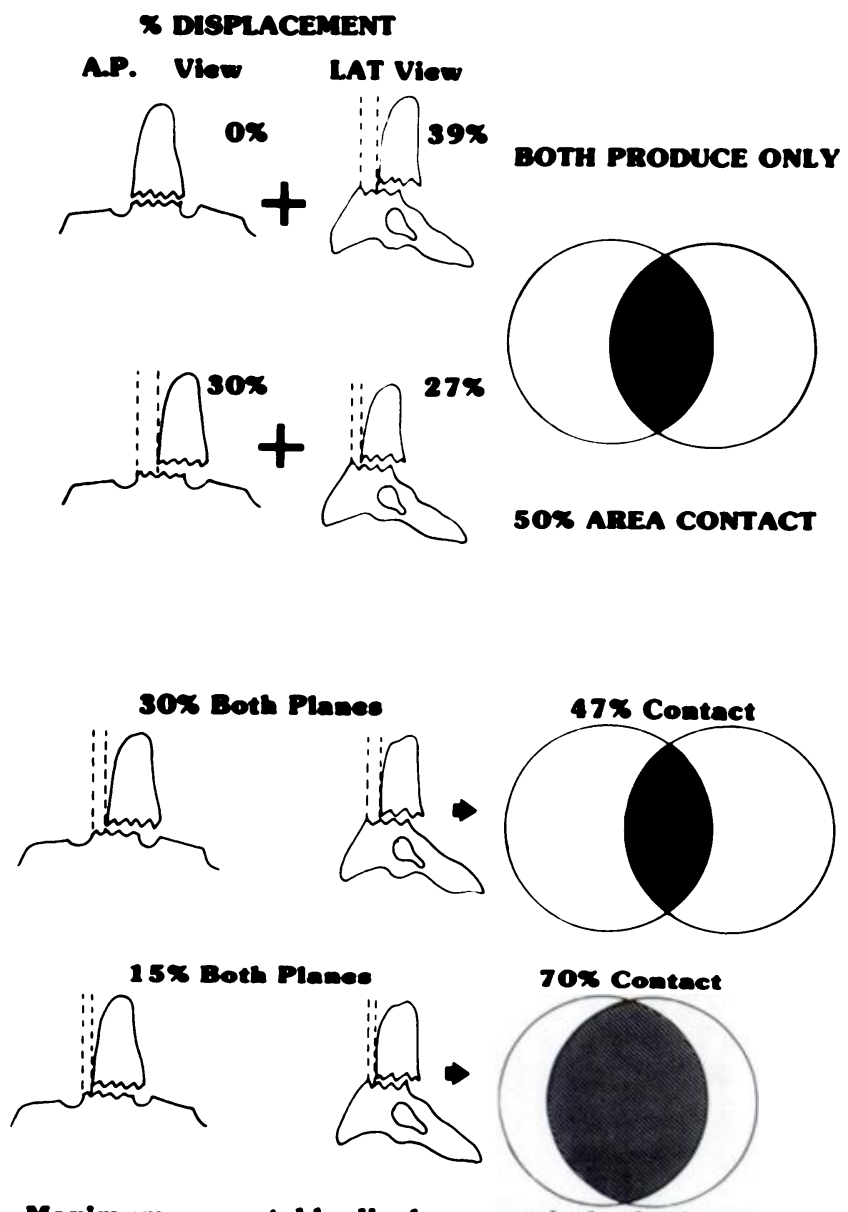

Maximum accoptable dieplacement in both planes: $20 x$ Surface contact: $63.9 \%$

Fig. 9

Diagram showing the area of contact in relation to displacement at the fracture site.

Immobilisation. Odontoid fractures must be immobilised adequately. Johnson et al. (1977) in a study of cervical orthoses, showed that a halo combined with a plastic body-vest allowed only four per cent of the normal flexion-extension movement of the whole cervical spine to occur, and that very little of this movement took place in the upper cervical region. It was also the most effective of all orthoses in controlling rotation, allowing only one per cent of the normal movement.

Blood supply. Experimental studies of the odontoid in dogs, whose vascular pattern is similar to that in man, suggest that the blood supply does not seem to influence fracture healing (Schatzker et al. 1975). 
Age. Schatzker et al. (1971) reported that the incidence of non-union appeared to be higher in patients over the age of 60 years. Schweigel (1979), however, in a series treated by a halo combined with a thoracic brace, found that advanced age did not prolong the time taken to achieve union.

\section{MATERIAL}

This paper reports a series of 23 odontoid fractures in adults, treated over a 10-year period in the Department of Orthopaedics and Traumatic Surgery at The Royal North Shore Hospital of Sydney. There were 17 men and six women. Five patients were referred late, that is, more than two weeks after injury. The average age of the men was 44 years, and that of the women 57 years. In 21 of the patients the cause was a blow to the head. Seventeen patients were injured in motor vehicle accidents; in two the cause was falling from a horse. Four patients, two of whom were inebriated at the time of the accidents, fell over in unrecorded circumstances. Most patients had bruising of the forehead and a short period of unconsciousness was common. All 23 fractures were displaced in the initial radiographs.

\section{TREATMENT}

Patients with recent fractures were treated either in a halo-cast* to control skull movement, or in a Minerva jacket. The cast extends only to the lower margin of the rib cage (Fig. 10) and must not rest on the pelvis; if it does there is a high risk that the screws of the halo may tear out of the skull when the patient sits.

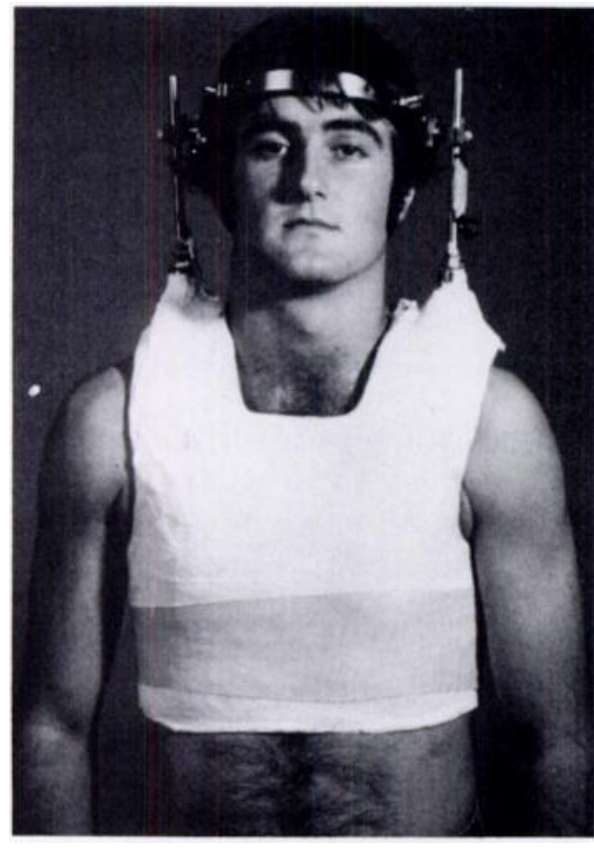

Fig. 10

Patient in a halo-cast.

* Twin Cities Low Profile Halo System supplied by De Puy Division of Boehringer Mannheim, 6 Byfield Road, North Ryde, New South Wales 2113, Australia.
Type 1 . The only example of a Type 1 injury healed after six weeks immobilisation in a halo-cast.

Type 2. Five Type 2 injuries were seen late, that is, more than two weeks after injury. Of the 11 recent fractures one was fused primarily because of an associated irreducible dislocation of $\mathrm{C} 2$ on $\mathrm{C} 3$, six were treated in halo-casts, three in Minerva jackets, and one in a SOMI $\dagger$ brace.

Type 3. There were six such fractures. Two were treated in halo-casts, two in Minerva jackets and one in a SOMI brace. One severely injured patient, who needed to be intubated for a long period, was given no external support.

\section{RESULTS}

Type 1 . The only Type 1 fracture was healed at six weeks. Type 2. Of the 11 Type 2 fractures one (already described) was fused primarily, and one (the patient treated in a SOMI brace) was lost to follow-up. The three patients treated in Minerva jackets all healed, but only four of the six treated in halo-casts united; in one of the two who did not unite, the fracture had redisplaced after the initial reduction (Figs 11 and 12). Thus, seven out of nine healed. The average duration of immobilisation was eight weeks.

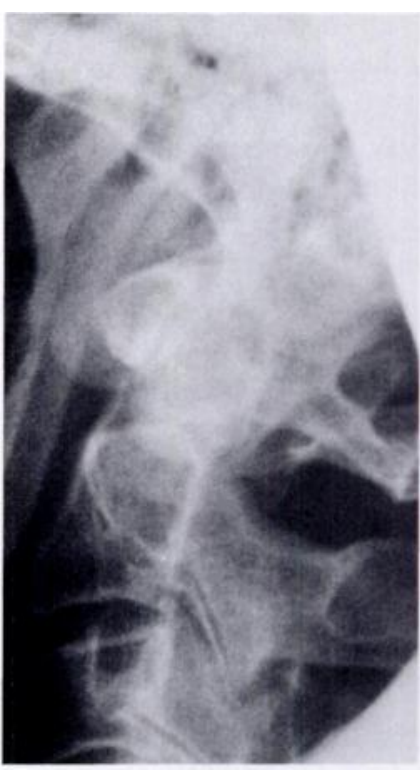

Fig. 11

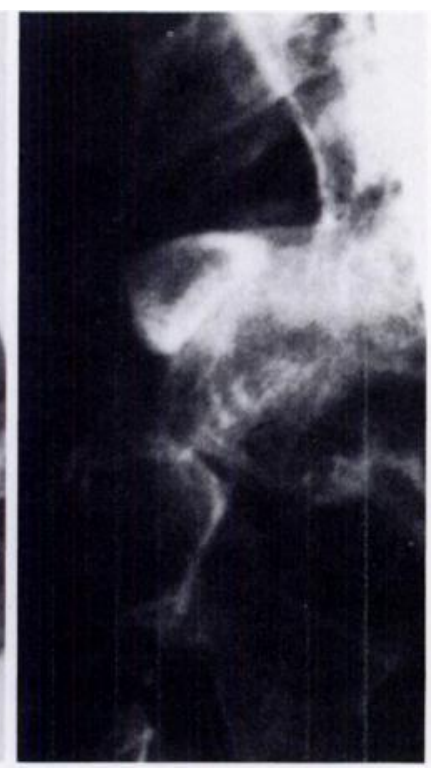

Fig. 12 Loss of position in a halo-cast.

Of the five "late" Type 2 fractures only one progressed to union. Two of the remainder required spinal fusion; the other two patients had less than five millimetres of anteroposterior shift on flexion-extension radiographs, and, as symptoms were minimal, fusion was not deemed necessary.

Type 3. All six such fractures were solidly united in an

+SOMI brace: Sterno-Occipito Mandibular Immobiliser supplied by US Manufacturing Company, Glendale, California, USA. 
average time of seven weeks. Thus our experience of Type 3 fractures confirms that of Anderson and D'Alonzo (1974) who reported that they healed well.

The overall healing rate of recent fractures was $\mathbf{8 7 . 5}$ per cent. All fractures which healed after immobilisation showed more than 66 per cent overlap of the fragments in the lateral radiographs and almost anatomical alignment in the anteroposterior views; thus there was a contact area of more than 60 per cent.

\section{DISCUSSION}

Both non-unions which occurred after halo-cast treatment were in fractures which, in the lateral radiographs taken after attempted reduction, were still displaced by at least 40 per cent; the contact area was thus less than 50 per cent. Had the overlap in the anteroposterior view been only 70 per cent, the contact area would have been less than 40 per cent (Fig. 9).

In the series reported by Blockey and Purser (1956) 13 fractures were not immobilised and all 13 failed to unite; six of the 10 treated by traction also did not unite; but all seven who were immobilised in plaster healed. Schweigel (1979) reported 13 unclassified odontoid fractures, treated in halo-thoracic braces, all of which eventually united. Clearly immobilisation is effective; skull traction is not, and may indeed be harmful. A striking example of the effectiveness of the halo-cast method is shown in Figures 13 and 14.

Our management of a recent fracture of the odontoid in an adult consists of early reduction followed by stabilisation in a halo-cast. Skull traction is never used. The halo-cast is well tolerated by male patients, and is easy to apply. It provides the best control of flexion, extension and rotation of the upper cervical spine (Johnson et al. 1977). Women tend to prefer a Minerva jacket, which also controls rotation effectively. Adequate reduction must be achieved, that is at least 70 per cent overlap of the fragments in a lateral radiograph, with near anatomical alignment in the anteroposterior view. This means that at least two-thirds of the fracture surfaces are in contact. There must be no gap at the fracture; if a gap of more than a few millimetres persists (Fig. 15), a good case can be made for $\mathrm{Cl}-2$ fusion as the primary treatment. Primary fusion is indeed considered appropriate whenever reduction is inadequate. Check radiographs should be taken at intervals of one or two weeks after the halo-cast has been applied, to ensure that loss of reduction is not missed.

In patients who present more than two weeks after injury, a sound case can be made for fusion as the primary

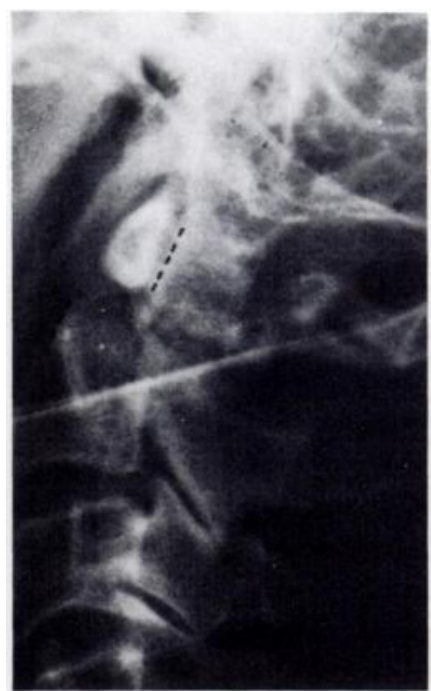

Fig. 13

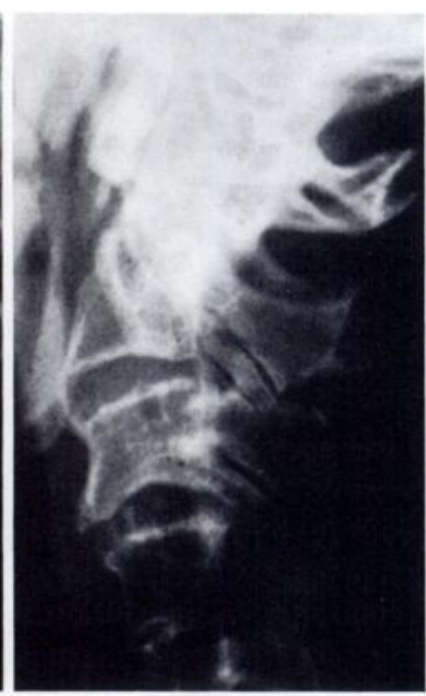

Fig. 14
Figure 13-This patient had a tetraparesis. Figure 14-After reduction and maintenance in a halo-cast, the fracture was solidly united at eight weeks and there was good neurological recovery.

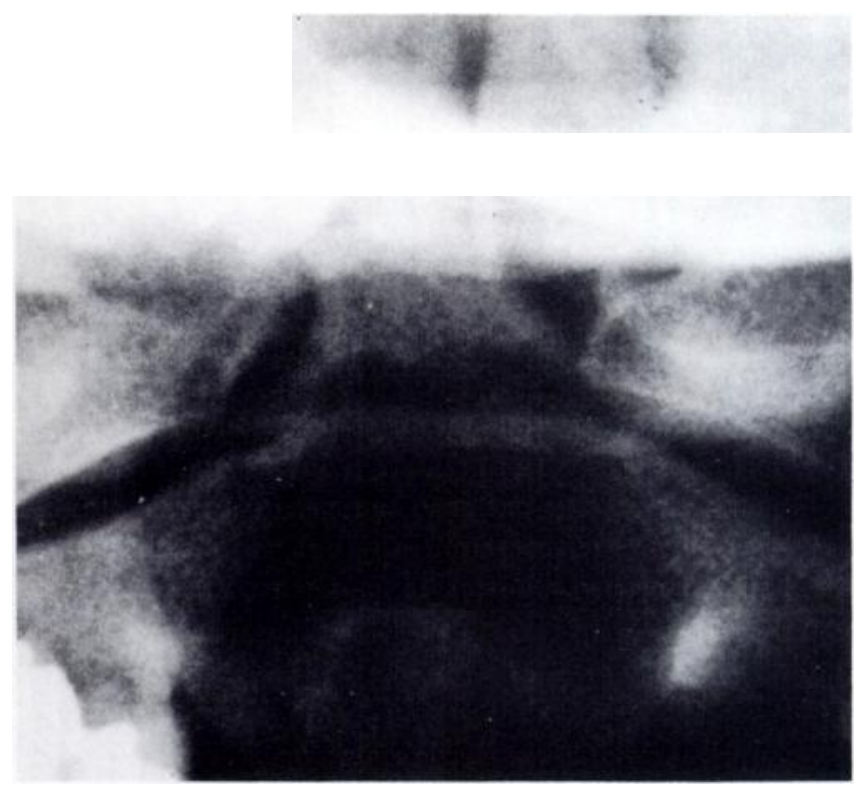

Fig. 15

A gap at the fracture site produced by traction in an adult.

treatment; four out of five late fractures in this present series did not unite. It is our current practice, in established non-union, to accept up to four millimetres of anteroposterior movement of the dens in flexion and extension radiographs, but fusion is advised if there is greater instability than this, any neurological deficit or pain. The decision is influenced by the patient's age and activities.

\section{REFERENCES}

Anderson LD, D'Alonzo RT. Fractures of the odontoid process of the axis. J Bone Joint Surg [Am] 1974:56-A:1663-74

Amyes EW, Anderson FM. Fracture of the odontoid process: report of sixty-three cases. Arch Surg 1956;72:377-93

Blockey NJ, Purser DW. Fractures of the odontoid process of the axis. J Bone Joint Surg [Br] 1956:38-B: 794-817.

Böhler J. Fractures of the odontoid process. J Trauma 1965;5:386-91.

Crooks F, Birkett AN. Fractures and dislocations of the cervical spine. Br J Surg 1944;31:252-65. 
Jefferson G. Fracture of the atlas vertebra : report of four cases and a review of those previously recorded. $B r J$ Surg 1920;7:407-22.

Jefferson G. Remarks on fractures of the first cervical vertebra. Br Med J 1927;2:153-7.

Johnson RM, Hart DL, Simmons EF, Ramsby GR, Southwick WO. Cervical orthoses: a study comparing their effectiveness in restricting cervical motion in normal subjects. J Bone Joint Surg [Am] 1977;59-A :332-9.

Mouradian WH, Fietti VG Jr, Cochran G van B, Fielding JW, Young J. Fractures of the odontoid : a laboratory and clinical study of mechanisms. Orthop Clin North Am 1978;9:985-1001.

Nachemson A. Fracture of the odontoid process of the axis: a clinical study based on 26 cases. Acta Orthop Scand 1959;29:185-217.

Plaut HF. Fractures of the atlas resulting from automobile accidents: a survey of the literature and report of 6 cases. AJR 1938;40:867-90.

Schatzker J, Rorabeck CH, Waddell JP. Fractures of the dens (odontoid process): an analysis of thirty-seven cases. J Bone Joint Surg [Br] $1971 ; 53-B: 392-405$.

Schatzker J, Rorabeck CH, Waddell JP. Non-union of the odontoid process: an experimental investigation. Clin Orthop 1975; 108 : 127-37.

Schweigel JF. Halo-thoracic brace management of odontoid fractures. Spine 1979;4:192-4. 